\title{
Bedingte Akzeptanz? Moralische und rechtsethische Dimensionen der Ablehnung künftiger Kinder
}

\section{Kommentar zum Artikel „Die Auswahl zukünftiger Kinder“ von Tatjana Tarkian}

\author{
Markus Rothhaar
}

Online publiziert: 15 . Juni 2020

(C) Der/die Autor(en) 2020

Zusammenfassung Im Kommentar zum Text von Tatiana Tarkian werden zwei Argumente der Verfasserin einer kritischen Prüfung unterzogen: Zum einen das Argument, dass parentale Tugenden einer Auswahl künftiger Kinder nicht entgegenstehen würde; zum anderen das Argument, dass eine negative Selektion aufgrund von Behinderung keinen diskriminierenden Charakter gegenüber (geborenen) Behinderten habe, wenn die negative Selektion ledgilich aufgrund verbreiteter diskriminierender Urteile in der Gesellschaft erfolge.

Der Text von Tatjana Tarkian (2020) behandelt die Frage, ob es gute Gründe geben könnte, die Freiheit zur „Auswahl künftiger Kinder“ einzuschränken. Dabei diskutiert die Verfasserin drei mögliche Argumente für eine solche Beschränkung der reproduktiven Freiheit, die sie alle zurückweist: Das Argument der parentalen Tugenden, das Argument der Diskriminierung Behinderter und das Argument des Wohlergehens des künftigen Kindes. Ich werde in diesem Kommentar der damit vorgegebenen Strukturierung folgen, mich aber auf die beiden ersten Argumente konzentrieren. Zunächst scheinen mir aber noch zwei Vorbemerkungen von Bedeutung:

Zum einen spricht die Verfasserin in ihrem Text etwas vage von „moralischen Gründen“, die für eine Beschränkung der Freiheit zur Auswahl künftiger Kinder sprechen könnten. Gerade vor dem Hintergrund der dezidiert liberalen Positionierung der Verfasserin erscheint diese Redeweise allerdings problematisch, denn in einem liberalen Rechtsverständnis reichen bloß „,moralische Gründe“ nicht unbedingt aus, um Einschränkungen der Handlungsfreiheit zu rechtfertigen. Vielmehr muss jeweils gezeigt werden, dass die Handlung, die untersagt oder eingeschränkt

M. Rothhaar ( $\square)$

Institut für Philosophie, Lehrgebiet Philosophie II, Fernuniversität Hagen, 58084 Hagen, Deutschland E-Mail: markus.rothhaar@fernuni-hagen.de 
werden soll, die Handlungsfreiheit der anderen auf eine Weise tangiert, die mit einer gleichen Aufteilung von Freiheitssphären unvereinbar ist. Hat man diese Differenzierung zwischen Moral und Rechtsethik einmal vorgenommen, kann es durchaus sein, dass es unterhalb der Schwelle der Rechtfertigung rechtlicher Verbote gute, auch allgemeinverbindliche moralische Gründe gegen eine Auswahl künftiger Kinder gibt. Zugleich wäre die Schwelle für rechtliche Einschränkungen oder Verbote wohl deutlich höher.

Zum anderen macht die Verfasserin eine sehr folgenschwere Voraussetzung, wenn sie die normative Bewertung von Schwangerschaftsabbruch und Präimplantationsdiagnostik mit dem Argument nicht diskutiert, dass es sich dabei um bloße Mittel der Auswahl künftiger Kinder handele, die als solche von der Frage nach der normativen Bewertung des Zwecks der Auswahl abgetrennt diskutiert werden könnten. Das erscheint schon deshalb wenig plausibel, da diejenige Auswahl künftiger Kinder, die Tarkian primär im Auge hat, kaum durch andere Mittel als Schwangerschaftsabbruch oder Präimplantationsdiagnostik bewerkstelligt werden kann. Wenn das der Fall ist, kann die Frage nach der moralischen und rechtsethischen Bewertung solcher Auswahl aber eigentlich nicht beantwortet werden, ohne die Frage nach der Zulässigkeit der Mittel und damit nach dem Status menschlicher Embryonen und Föten zu behandeln.

Die Folgen des Ausblendens der Statusfrage zeigen sich sehr deutlich im Hinblick auf den ersten Einwand, den Tarkian diskutiert, nämlich anhand des Einwandes der parentalen Tugenden. Tarkian stellt in diesem Zusammenhang fest, dass es zwar zum Kernbestand elterlicher Tugenden gehöre, ,seine Hinwendung zum Kind nicht an Bedingungen zu knüpfen und den Platz des Kindes in der Familie nicht von seinen spezifischen Merkmalen oder seiner Erfüllung elterlicher Erwartungen abhängig zu machen“, dass diese Pflicht aber lediglich für aktuale, nicht für bloß „künftige“ oder ,potentielle“ Kinder gelte. Damit macht die Verfasserin offenbar die implizite Voraussetzung, dass es sich bei einem menschlichen Embryo bzw. Fötus (noch) nicht um ein moralisch oder gar rechtlich zu berücksichtigendes Wesen handelt. Embryonen oder Föten kommen mithin bei Tarkian nicht als Wesen in den Blick, denen gegenüber eventuell auch moralische oder sogar rechtliche Pflichten bestehen könnten, und sei es in abgestufter Form, sondern lediglich als gewissermaßen ,aktual nicht existierende Kinder".

Der zweite Einwand gegen die Auswahl künftiger Kinder, den Tarkian zu entkräften versucht, besteht darin, dass die Entscheidung gegen Kinder, die mutmaßlich Behinderungen oder Beeinträchtigungen aufweisen würden, ein diskriminierendes Urteil gegen (auch und gerade geborene) Behinderte zum Ausdruck bringe und fortschreibe. In der Diskussion dieses Einwandes behandelt die Verfasserin dann aber fast ausschließlich die Frage, wann die Entscheidung gegen ein behindertes Kind tatsächlich Ausdruck von Selbstbestimmung ist und wann sie lediglich aufgrund verbreiteter diskriminierender Urteile in der Gesellschaft getroffen würde. Dabei ist die Verfasserin offenbar der Auffassung, dass eine Entscheidung gegen ein behindertes Kind nur dann eine Diskriminierung zum Ausdruck bringt, wenn sie nicht selbstbestimmt getroffen wurde, sondern von verbreiteten diskriminierenden Urteilen in der Gesellschaft beeinflusst wurde. Diese Art, das Problem zu behandeln, ist aber wenig überzeugend. Zum einen beruht sie auf einer hypertrophen Vorstellung 
von Selbstbestimmung und Autonomie (die leider in der Medizinethik weit verbreitet ist), wonach nur bindungs- und kontextlose Entscheidungen als selbstbestimmt gelten können. Wichtiger ist aber noch ein anderer Punkt: Für die Frage, ob eine bestimmte Entscheidung diskriminierenden Charakter besitzt (hier konkret: ob sie das Urteil impliziert, dass das Leben eines/einer Behinderten nicht lebenswert bzw. das Leben mit ihm/ihr nicht zumutbar sei), ist ja - nicht zuletzt aus der Sicht behinderter Menschen - gerade von Bedeutung, was in dieser Entscheidung inhaltlich zum Ausdruck kommt. Ob die Entscheidung dagegen unter dem Einfluss gesellschaftlicher Vorurteile zustande gekommen ist oder ob der Mensch, der sie trifft, sie aufgrund eigener Überlegungen getroffen hat, ist genau dafür nicht relevant. Ja eigentlich wäre letzteres sogar in einer bestimmten Hinsicht noch problematischer als ersteres, da der/die Entscheidende nicht einmal davon entlastet wäre, selbst zu dem diskriminierenden Urteil gekommen zu sein. Insofern bleibt der Einwand des diskriminierenden Charakters entsprechender Urteile und Entscheidungen durchaus bestehen und wird von Tarkian keineswegs widerlegt.

Funding Open access funding provided by FernUniversität in Hagen (3099).

Open Access Dieser Artikel wird unter der Creative Commons Namensnennung 4.0 International Lizenz veröffentlicht, welche die Nutzung, Vervielfältigung, Bearbeitung, Verbreitung und Wiedergabe in jeglichem Medium und Format erlaubt, sofern Sie den/die ursprünglichen Autor(en) und die Quelle ordnungsgemäß nennen, einen Link zur Creative Commons Lizenz beifügen und angeben, ob Änderungen vorgenommen wurden.

Die in diesem Artikel enthaltenen Bilder und sonstiges Drittmaterial unterliegen ebenfalls der genannten Creative Commons Lizenz, sofern sich aus der Abbildungslegende nichts anderes ergibt. Sofern das betreffende Material nicht unter der genannten Creative Commons Lizenz steht und die betreffende Handlung nicht nach gesetzlichen Vorschriften erlaubt ist, ist für die oben aufgeführten Weiterverwendungen des Materials die Einwilligung des jeweiligen Rechteinhabers einzuholen.

Weitere Details zur Lizenz entnehmen Sie bitte der Lizenzinformation auf http://creativecommons.org/ licenses/by/4.0/deed.de.

\section{Literatur}

Tarkian T (2020) Die Auswahl zukünftiger Kinder. Z Ethik Moralphilosophie. https://doi.org/10.1007/ s42048-020-00068-2 